\title{
Criterios de predicción de inestabilidades plásticas en procesos de conformado en caliente. (Parte I: Revisión teórica) $)^{(\cdot)}$
}

\author{
A. Al Omar* y J.M. Prado**
}

Resumen

\begin{abstract}
Los procesos de conformado de materiales inducen frecuentemente altos grados de deformación, a grandes velocidades de deformación, e imponen modos de solicitación multiaxiales y muy complejos. Dichos procesos quedan esencialmente limitados por la aparición y desarrollo de inestabilidades plásticas. Éstas pueden ser la causa directa de la propagación rápida de una fisura, que puede dar lugar a una rotura final. La complejidad de los modos de deformación y la intervención simultanea de varios parámetros han llevado a varios investigadores a desarrollar diversos criterios, con diferentes planteamientos, para predecir la aparición de defectos y optimizar los parámetros de control en los procesos de conformado. El presente trabajo pretende exponer, a modo de revisión, algunos criterios ampliamente usados en la literatura para la predicción de las inestabilidades plásticas durante el conformado de materiales. Se ha estimado oportuno dividir la materia del trabajo en dos partes: una primera parte presenta los criterios fenomenológicos de predicción de las inestabilidades plásticas, basados en la observación descriptiva de los fenómenos microscópicos de la deformación (endurecimiento por deformación y sensibilidad a la velocidad de deformación), luego se analizan los criterios continuos basados en los principios de máxima velocidad de producción de entropía de la termodinámica irreversible aplicada a la mecánica continua de la fluencia plástica en grandes deformaciones. Asimismo, se comenta una discusión bibliográfica entablada entre varios autores referida al fundamento físico del Modelo Dinámico de Materiales. En la segunda parte, se lleva a cabo un estudio comparativo para caracterizar la inestabilidad plástica durante un proceso de conformado en caliente de un acero microaleado de medio carbono usando criterios continuos y fenomenológicos.
\end{abstract}

Palabras clave

Conformado en caliente; Criterios de Inestabilidades plásticas; Modelo dinámico de materiales; Localización de la fluencia.

\section{Criteria for prediction of plastic instabilities for hot working processes. (Part I: Theoretical review)}

\begin{abstract}
Hot working processes often induce high levels of deformation at high strain rates, and impose very complex multiaxial modes of solicitation. These processes are essentially limited by apparition and development of plastic instabilities. These may be the direct cause of rapid crack propagation, which lead to a possible final rupture. The complexity of deformation modes and the simultaneous intervention of several parameters have led many researchers to develop various criteria, with different approaches, to predict the occurrence of defects and to optimize process control parameters. The aim of the present paper is to summarize the general characteristics of some instability criteria, widely used in the literature, for the prediction of plastic instabilities during hot working. It was considered appropriate to divide the work into two parts: part I presents the phenomenological criteria for the prediction of plastic instabilities, based on descriptive observation of microscopic phenomena of the deformation (strain hardening and strain rate sensitivity), and discusses the continuum criteria based on the principle of maximum rate of entropy production of irreversible thermodynamics applied to continuum mechanics of large plastic flow. Also, this part provides a bibliographical discussion among several authors with regard to the physical foundations of dynamic materials model. In part II, of the work, a comparative study has been carried out to characterize the flow instability during a hot working process of a medium carbon microalloyed using phenomenological and continuum criteria.
\end{abstract}

Keywords Hot working; Plastic instabilities criteria; Dynamic material model; Flow localization.

\footnotetext{
(•) Trabajo recibido el día 16 de julio de 2009 y aceptado en su forma final el día 19 de noviembre de 2009.

* Departamento de Ingeniería Mecánica, EPSEM, Universidad Politécnica de Cataluña, Av. Bases de Manresa 6173,08240 Manresa, Barcelona, España.

${ }^{* \star}$ Centro Tecnológico de Manresa, Av. Bases de Manresa 1, 08240 Manresa, Barcelona, España.
} 


\section{INTRODUCCIÓN}

Hoy en día, el mercado internacional plantea un serio reto a las empresas de fabricación mecánica ya que los procesos de conformado se deben llevar a cabo de la forma más económica posible; garantizando la calidad requerida por el producto conformado a lo largo de su vida. Todo ello, ha forzado a los fabricantes a acortar los tiempos de diseño y, al mismo tiempo, a no correr riesgos en los procesos de desarrollo y fabricación del producto.

La competitividad y la rentabilidad económica de todo proceso de conformado, como es bien sabido, depende del control de sus parámetros y de la correcta elección de sus diferentes etapas y secuencias. A pesar de los esfuerzos dedicados a la optimización de dichos procesos, la habilidad de transformación de materiales metálicos brutos en piezas útiles se ve limitada por la aparición de inestabilidades plásticas (fluencia localizada, formación de cavidades, bandas de cizalladura, fisuración intergranular, fisuración en cuña, fluencia localizada, etc.) que van en detrimento de la conformabilidad del material sometido a procesos de conformado ${ }^{[1-15]}$. Dichas inestabilidades pueden ser la causa directa de la propagación rápida de una fisura, dando así origen a un fenómeno de fractura rápida y causando fallas catastróficas. Por ello, el estudio de los criterios que predicen la aparición del fallo por inestabilidades plásticas es muy importante, no solo desde un punto de vista científico e ingenieril sino también desde un punto de vista económico.

Predecir la aparición de las inestabilidades plásticas en un proceso de conformado resulta extremadamente difícil, debido a los efectos y a las interacciones no lineales entre los parámetros del proceso y las propiedades del material conformado, y a la complejidad de los mecanismos físicos y microestructurales que intervienen durante el conformado tales como: la anisotropía inicial e inducida, las heterogeneidades de textura, el calentamiento adiabático, la recristalización dinámica, el crecimiento de grano, etc. ${ }^{[1,3,4 \text { y }}$ 6]. Por tanto, para una descripción adecuada del comportamiento de fluencia en caliente no sólo hay que considerar la influencia de variables físicas, tales como temperatura, velocidad de deformación, etc., sino también factores microestructurales como la densidad de defectos y su movilidad, la formación de subestructuras, tamaño de grano, etc.

\section{ANÁLISIS DE CRITERIOS FENOME- NOLÓGICOS Y TERMODINAMICOS CONTINUOS PARA LA PREDICCIÓN DE LA FLUENCIA LOCALIZADA}

En el diseño de los procesos de conformado, es necesario entender los modos de deformación que intervienen y dominan los parámetros de control de dichos procesos, para poder predecir con exactitud la geometría final de la pieza conformada y optimizar sus propiedades mecánicas. Por lo tanto, el diseñador del proceso de fabricación debe tener muy claro el criterio que tiene que adoptar, en el proceso de conformado, para evitar la aparición de inestabilidades plásticas y producir productos finales con microestructuras y propiedades controladas.

En este trabajo, se hace una breve revisión de algunos criterios de inestabilidad plástica usados, en la literatura, para la predicción de las inestabilidades plásticas durante el conformado de materiales metálicos. A grandes rasgos, se pueden distinguir dos grandes tendencias en el desarrollo de dichos criterios:

- Criterios fenomenológicos mecánicos basados en una observación descriptiva y una interpretación mecánica de los fenómenos microscópicos de la deformación (endurecimiento por deformación y sensibilidad a la velocidad de deformación). Estos criterios son deducidos a partir de hipótesis de comportamiento y reflejan el mecanismo de la inestabilidad plástica. Teniendo en cuenta las indicaciones cualitativas de la física de sólidos y de la termodinámica, se puede llegar a obtener criterios sencillos y realistas.

- Criterios continuos termodinámicos basados en los principios de máxima velocidad de producción de entropía de la termodinámica irreversible aplicada a la mecánica continua de la fluencia plástica en grandes deformaciones. La aproximación termodinámica utiliza un medio continuo homogenizado equivalente al medio real, y representa los fenómenos físicos mediante variables macroscópicas. Éstas permiten optimizar los parámetros de control y definir los dominios óptimos de deformación en los mapas de procesado, donde se evitan las inestabilidades plásticas y se consigue la máxima eficiencia energética.

Para analizar los criterios de inestabilidad plástica, los siguientes parámetros adimensionales se determinan a partir de las curvas de fluencia a diferentes temperaturas y velocidades de deformación:

El parámetro de sensibilidad a la velocidad de deformación:

$$
m=\left(\frac{\partial \ln \sigma}{\partial \ln \dot{\varepsilon}}\right)=\frac{\dot{\varepsilon}}{\sigma}\left(\frac{\partial \sigma}{\partial \dot{\varepsilon}}\right)_{\varepsilon}
$$

El coeficiente de endurecimiento: 


$$
\gamma=\frac{1}{\sigma} \frac{\partial \sigma}{\partial \varepsilon}
$$

El parámetro de la localización de la fluencia ${ }^{[16]}$ :

$$
\alpha=\frac{\gamma-1}{m}
$$

El parámetro de sensibilidad a la temperatura de la tensión de fluencia:

$$
s=\left[-\frac{1}{T} \frac{\partial(\ln \sigma)}{\partial(1 / T)}\right]_{\varepsilon, \dot{\varepsilon}}
$$

El parámetro de la eficiencia de disipación de energía $[5$ y 7$]$ :

$$
\eta=\frac{J}{J_{\max }}=\frac{P-G}{J_{\max }}=\left(2-\frac{2 G}{P}\right)=\frac{2 J}{P}
$$

donde, la energía total disipada por unidad de volumen:

$$
P=G+J=\sigma \cdot \dot{\varepsilon}
$$

El contenido disipado, $\mathrm{G}$, para una temperatura y una deformación dada:

$$
G=\int_{0}^{\dot{\varepsilon}} \sigma \cdot d \dot{\varepsilon}
$$

El co-contenido disipador, $J$ :

$$
J=\int_{0}^{\sigma} \dot{\varepsilon} \cdot d \sigma
$$

El co-contenido disipador máximo:

$$
J_{\max }=\left(\frac{\sigma \cdot \dot{\varepsilon}}{2}\right)=\frac{P}{2}
$$

\subsection{Criterios fenomenológicos mecánicos}

Durante un proceso de conformado, la transformación de un material metálico se debe, generalmente, a un estado de tensiones y deformaciones muy complejo e intenso. La deformación empieza siendo heterogénea y, a medida que va avanzando, aparecen fenómenos de inestabilidad plástica. Esto se debe, probablemente, a las maneras de aplicación de los esfuerzos sobre el material conformado y, también, a las condiciones de fricción y a los efectos de los mecanismos microestructurales ${ }^{[2}$ y 6$]$.

La manifestación más común de la inestabilidad plástica es la aparición de la deformación localizada, durante la conformación volumétrica, en forma bandas de cizalladura (regiones de cizalladura localizada cruzando algunos granos y observables a escala macroscópica) ${ }^{[16]}$. Debido a sus nefastos efectos, el origen de las bandas de cizalladura ha sido tema de mucho interés para numerosos investigadores ${ }^{[17-23]}$. Es bien conocido que estas bandas pueden desarrollarse a velocidades de deformación relativamente bajas (procesos isotérmicos de forja), a velocidades de deformación intermedias (procesos convencionales de conformado de materiales) y a altas velocidades de deformación (corte de metales y balística).

A pesar de su amplia aparición en los procesos de conformado, una descripción exacta y cuantitativa de la localización de la fluencia y de los factores que la gobiernan está todavía por desarrollar. La mayoría de las correlaciones existentes entre la presencia de las bandas de cizalladura y las propiedades del material conformado se basan en la estimación de la deformación correspondiente a la tensión de inestabilidad o al máximo de la tensión de fluencia ${ }^{[20 \text { y } 21]}$. Otros modelos ${ }^{[24}$ y 25] toman en cuenta los efectos de la deformación y del endurecimiento sobre la localización de la fluencia y tratan el problema como si fuese un proceso y no como un evento específico. Estos modelos intentaron describir el fenómeno de la localización de la deformación en el conformado de materiales bajo distintos modos de carga, asumiendo la presencia de una imperfección inicial, geométrica o de deformación y midiendo su crecimiento relativo al resto de la pieza conformada, para conseguir un equilibrio en las dos regiones. De esta manera, el grado exacto de la localización de la fluencia, conocido como el límite de conformado, ha podido ser evaluado ${ }^{[24-29]}$

\subsubsection{El criterio de Considère en tracción y compresión}

Desde que Considère publicó su trabajo clásico sobre la estricción, en $1885^{[30]}$, se reconoció claramente que, en tracción, la localización de la fluencia empieza cuando la velocidad de endurecimiento no es lo suficientemente alta como para compensar la disminución en la sección transversal. En torsión ${ }^{[28]}$, se demostró que 
la velocidad de endurecimiento debe anularse al no cambiarse el área de la sección deformada mientras que en ensayos de compresión ${ }^{[26]}$, debe ser suficientemente negativa para compensar la velocidad de crecimiento del área transversal. Así, se consideraba que la localización de la fluencia no puede manifestarse hasta que la velocidad de endurecimiento alcance un valor crítico.

La premisa básica del criterio de Considère ${ }^{[30]}$ se basa en que la estricción, como manifestación de inestabilidad plástica durante el ensayo de tracción, se inicia, generalmente, cuando se alcanza la carga máxima: el endurecimiento por deformación produce un incremento de la tensión de fluencia (debido a la disminución del área de la sección transversal de la probeta) más grande que la capacidad del material para soportar la carga. De esta manera, un material plástico ideal, en el cual no tiene lugar ningún endurecimiento por deformación, podría hacerse inestable en tracción e iniciarse la estricción tan pronto como empezase la fluencia.

El criterio de Considère, llamado también criterio de carga máxima, supone que la condición de aparición de la inestabilidad se satisface cuando la carga aplicada, F, pasa por un máximo. Dicha condición se escribe:

$$
\partial F=(\sigma \partial A+A \partial \sigma)=0
$$

donde, $F, \sigma$ y A son la carga, tensión y sección del área transversal, respectivamente.

De la constancia de volumen se deduce:

$$
\begin{gathered}
\frac{\partial V}{V}=\frac{\partial L}{L}+\frac{\partial A}{A}=0 \\
\frac{\partial \sigma}{\sigma}=\frac{\partial L}{L}=-\frac{\partial A}{A}=\partial \varepsilon=\frac{\partial e}{1+e}
\end{gathered}
$$

donde, $L$ es la longitud de la probeta.

Así, suponiendo que las deformaciones plásticas son independientes del tiempo, según Considère, la inestabilidad plástica se alcanzará cuando se verifique el criterio siguiente:

$$
\frac{\partial \sigma}{\partial \varepsilon}=\sigma
$$

La ecuación (13.I) indica que, en un ensayo de tracción uniaxial, el material se deformará progresivamente hasta alcanzar el punto donde $\frac{\partial \sigma}{\partial \varepsilon}=\sigma$, a partir del cual se producirá la estricción local con una deformación para la cual la pendiente de la curva tensión verdadera deformación verdadera es igual a la tensión verdadera correspondiente a esa deformación. La sección geométrica del cuello de la estricción se irá reduciendo hasta que la carga medida empiece a disminuir y se produzca la fractura.

Cuando la carga alcanza su máximo, se define la deformación crítica, $\varepsilon_{c^{*}}$. Si el comportamiento del material obedece a una ley potencial de tipo Hollomon: $\sigma=K \varepsilon^{n}$, es posible determinar, fácilmente, la deformación correspondiente a la estricción local:

$$
\frac{\partial \sigma}{\partial \varepsilon}=\sigma=K \varepsilon^{n}=n K \varepsilon^{n-1}
$$

Por tanto, la deformación de la estricción es numéricamente igual al coeficiente de endurecimiento por deformación, $n\left(\varepsilon_{c}=n\right)$.

Considère propuso, también, una manera gráfica para determinar la deformación de inicio de la inestabilidad plástica. Este método alternativo utiliza la definición de la deformación ingenieril, e:

$$
\varepsilon=\ln (1+e)
$$

o bien:

$$
\partial \varepsilon=\frac{\partial e}{1+e}
$$

De esta manera, una forma alternativa para establecer la condición de inestabilidad es:

$$
\frac{\partial \sigma}{\partial e}=\frac{\sigma}{1+e}
$$

En la figura 1, el punto A, de la curva $\sigma$ - e, representa una deformación negativa de valor absoluto, 1. Una línea trazada desde el punto $A(\varepsilon=-1, \sigma=0)$ y tangente a la curva $\sigma$ - e indicará el punto de carga máxima porque, de acuerdo con la ecuación (17.I), la pendiente en este punto es $\frac{\sigma}{1+e}$. La tensión en este punto es la tensión verdadera con carga máxima.

Aunque el criterio clásico de Considère se basó en la deformación ingenieril, puede ser, también, desarrollado en términos de deformación verdadera. Esto, se hace representando $\sigma$ y $\frac{\partial \sigma}{\partial e}$ en función de $\varepsilon$, o bien introduciendo el coeficiente de endurecimiento, $\gamma$ (Fig. 2). En la primera aproximación, el 


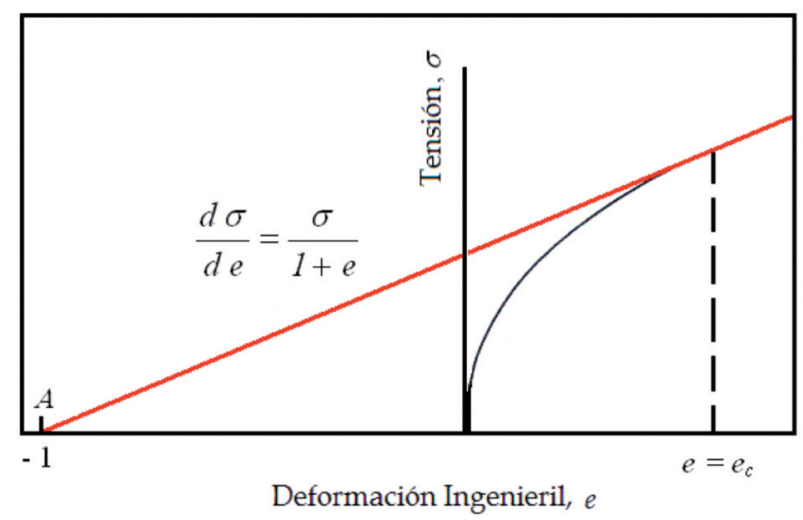

Figura 1. Aplicación del "criterio de Considère" para determinar el punto de carga máxima sobre la curva tensión verdadera-deformación ingenieril en un ensayo de tracción

Figure 1. Application of "Considère criterion" for determination of the nominal strain at maximum load in a tensile test.

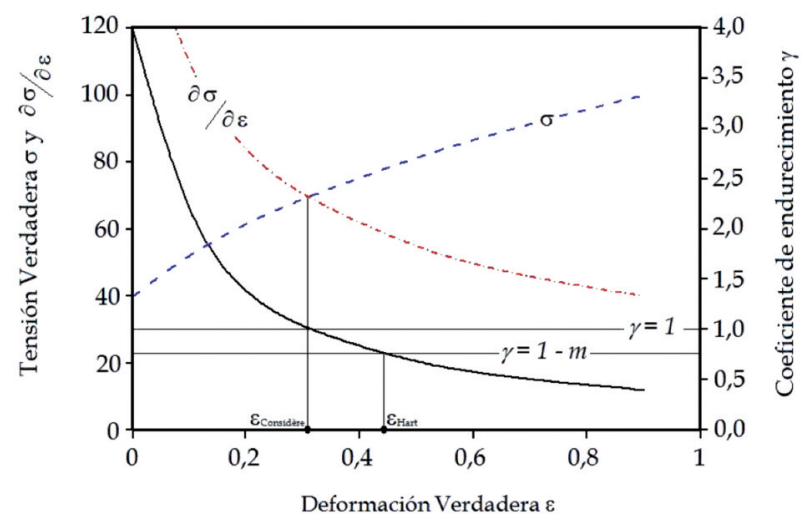

Figura 2. Evolución de $\sigma, \partial \sigma / \partial \varepsilon$ y $\gamma$ con la deformación e durante un ensayo de tracción ${ }^{[24]}$.

Figure 2. The dependence of $\sigma, \partial \sigma / \partial \varepsilon$ and $\gamma$ on strain e during a tensile test ${ }^{[24]}$.

inicio de la inestabilidad se define por la condición expresada en la ecuación (13.I), y en la segunda expresada por:

$$
\gamma-1=0
$$

Generalmente, el criterio de Considère no puede aplicarse en un ensayo de compresión ya que una particularidad muy importante y específica del ensayo de compresión es el aumento del área de la sección de la probeta. Dicho aumento hace incrementar la velocidad de endurecimiento. Sin embargo, cuando un ensayo de compresión se realiza a alta temperatura, varios mecanismos convencionales de endurecimiento se vuelven inestables, y aparecen los de ablandamiento por fluencia y los de inestabilidad plástica. En este caso, si la velocidad de ablandamiento por fluencia supera la del incremento de la sección transversal, una carga máxima será alcanzada, seguida de una carga mínima. Esta condición se representa en la figura 3 a), donde se puede observar que $\sigma$ y e son, ambas, negativas. También, en la figura $3 \mathrm{~b}$ ), se ilustra las condiciones expresadas en las ecuaciones (13.I) y (18.I) donde $\gamma-1$ es negativo para una fluencia estable.

\subsubsection{El criterio de Hart}

El análisis de Considère es aplicable, solamente, a los materiales insensibles a la velocidad de deformación. Backofen et al. ${ }^{[31]}$ fueron de los primeros en tratar de cuantificar el efecto de la sensibilidad a la velocidad de deformación. Posteriormente, Hart ${ }^{[32]}$, en uno de los primeros análisis del comportamiento de superplasticidad, desarrolló un análisis más general y unificado del ensayo de tracción. El punto de partida del análisis de Hart es la ecuación (16.I) y asumiendo que la ecuación constitutiva del comportamiento del material deformado incluye el endurecimiento por deformación y la sensibilidad a la velocidad de deformación, $\sigma=\sigma(\varepsilon, \dot{\varepsilon})$.

Puesto que:

$$
\dot{\sigma}=\frac{d \sigma}{d t}=\frac{1}{d t}\left[\left(\frac{\partial \sigma}{\partial \varepsilon}\right)_{\dot{\varepsilon}} d \varepsilon+\left(\frac{\partial \sigma}{\partial \dot{\varepsilon}}\right)_{\varepsilon} d \dot{\varepsilon}\right\rfloor
$$

es decir;

$$
\dot{\sigma}=\left[\left(\frac{\partial \sigma}{\partial \varepsilon}\right)_{\dot{\varepsilon}} \dot{\varepsilon}+\left(\frac{\partial \sigma}{\partial \varepsilon}\right)_{\varepsilon} \ddot{\varepsilon}\right]
$$

Por lo tanto,

$$
\dot{F}=\frac{d F}{d t}=\frac{(\sigma d A+A d \sigma)}{d t}=\sigma \dot{A}+A \dot{\sigma}=0
$$

Dividiendo la ecuación (21.I) por F, se obtiene:

$$
\frac{\dot{F}}{F}=\frac{(\sigma \dot{A}+A \dot{\sigma})}{\sigma A}=\frac{\dot{A}}{A}+\frac{\dot{\sigma}}{\sigma}
$$


Deformación Ingenieril, $e$
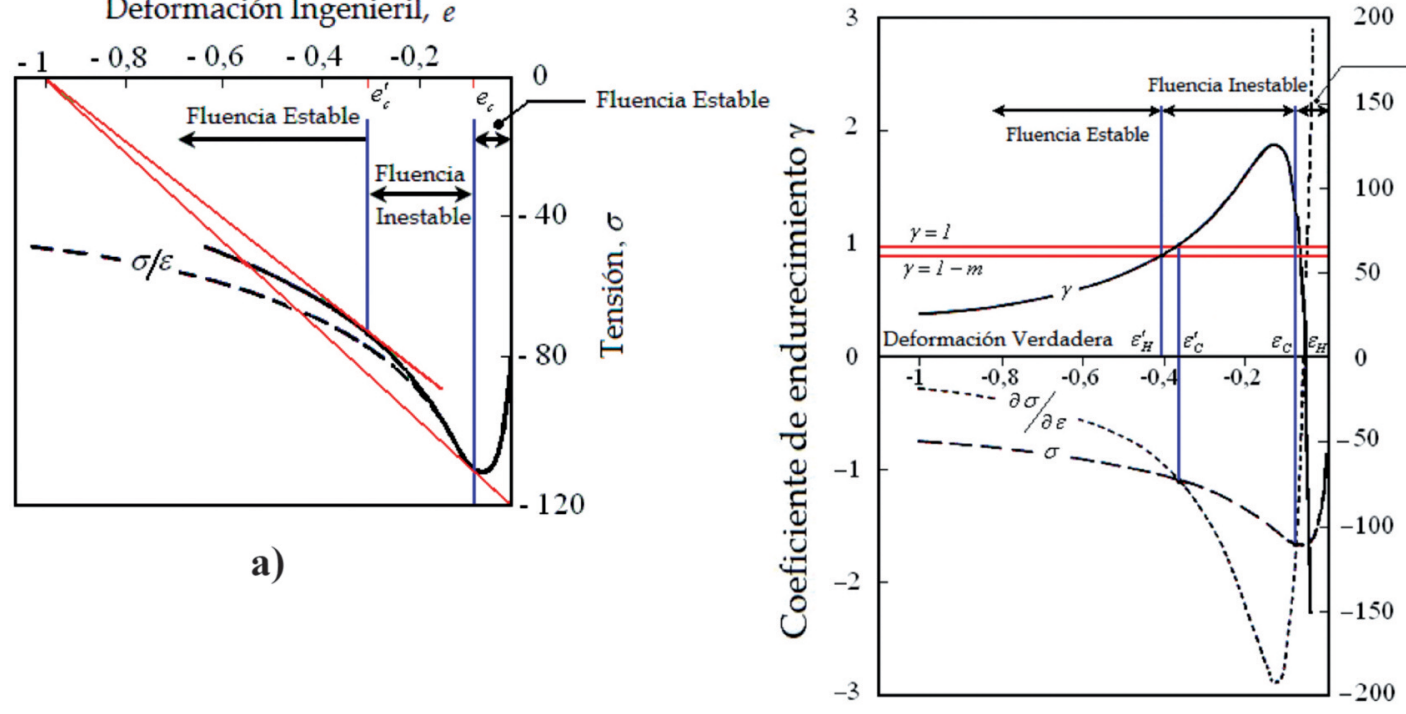

Fluencia Estable

a)

b)

Figura 3. a) Representación típica de un ensayo de compresión de un material que sufre un ablandamiento por fluencia ${ }^{[26]}$. El "criterio de Considère" modificado se usa para definir el inicio y el fin de la inestabilidad plástica, en la curva de fluencia tensión verdadera - deformación ingenieril $(\sigma-e)$, mediante las deformaciones ingenieriles críticas y $\mathrm{e}_{\mathrm{c}} \mathrm{y} \mathrm{e}_{\mathrm{c}}^{\prime}$. La curva tensión verdadera-deformación verdadera $(\sigma-\varepsilon)$ se representa en línea discontinua para poder comparar las dos curvas; b) Evolución de $\sigma, \partial \sigma / \partial \varepsilon$ y $\gamma$ con la deformación verdadera e durante un ensayo de compresión de un material que sufre ablandamiento por fluencia[26]. Es importante hacer notar que la deformación de Hart, $\varepsilon_{\mathrm{H}}$, para el inicio de la fluencia localizada precede la deformación de Considère, $\varepsilon_{c}$, para el inicio de la localización sensible a la velocidad de deformación. Es interesante, también, destacar que la deformación de Considère, $\varepsilon_{\mathrm{c}}^{\prime}$, para el fin de la fluencia inestable precede la de Hart, $\varepsilon_{\mathrm{H}^{\prime}}$.

Figure 3- a) A typical representation for compression test for a flow softening material[26]. A modified "Considère criterion" is used to define the onset and end of load instability on the true stress engineering strain flow curve $(\sigma-e)$. The true stress true strain curve $(\sigma-e)$ is given in broken line for purposes of comparison; b) The evolution of $\sigma, \partial \sigma / \partial \varepsilon$ and $\gamma$ on true strain $\varepsilon$ during the compression test of a flow softening material[26]. Note that the Hart strain $\varepsilon_{H}$ for the onset of flow localization precedes the "Considère" strain $\varepsilon_{c}$ for the onset of strain rate localization. It is also of interest that the "Considère" strain $\varepsilon_{c}^{\prime}$ for end of unstable flow precedes that of Hart strain $\varepsilon^{\prime}{ }_{H}$.

Sustituyendo por su expresión de la ecuación (20.I) en la (22.I), se tiene:

$$
\frac{\dot{F}}{F}=\frac{\dot{A}}{A}+\frac{1}{\sigma} \frac{\partial \sigma}{\partial \varepsilon} \dot{\varepsilon}+\frac{1}{\sigma} \frac{\partial \sigma}{\partial \dot{\varepsilon}} \ddot{\varepsilon}
$$

Sabiendo que $\gamma=\frac{1}{\sigma} \frac{\partial \sigma}{\partial \varepsilon}$ y $m=\left(\frac{\partial \ln \sigma}{\partial \ln \dot{\varepsilon}}\right)=\frac{\dot{\varepsilon}}{\sigma}\left(\frac{\partial \sigma}{\partial \dot{\varepsilon}}\right)_{\varepsilon}$ la ecuación (23.I) se transformaría en:

$$
\frac{\dot{F}}{F}=\frac{\dot{A}}{A}+\gamma \dot{\varepsilon}+\frac{m}{\dot{\varepsilon}} \ddot{\varepsilon}
$$

Respetando la constancia de volumen: $A L=$ Constante, es fácil demostrar que:

$$
\dot{\varepsilon}=\frac{\dot{L}}{L}=-\frac{\dot{A}}{A}
$$

Diferenciando la ecuación anterior con respecto del tiempo, se obtiene la siguiente expresión:

$$
\ddot{\varepsilon}=\frac{\partial \dot{\varepsilon}}{\partial t}=\frac{\ddot{L}}{L}-\left(\frac{\dot{L}}{L}\right)^{2}=-\frac{\ddot{A}}{A}+\frac{\dot{A}^{2}}{A}
$$

Usando las ecuaciones (25.I) y (26.I), la ecuación (24.I) se reduce a:

$$
\frac{\dot{F}}{F}=\frac{\dot{A}}{A}(1-\gamma-m)+\frac{\ddot{A}}{\dot{A}} m
$$


o bien:

$$
\frac{\dot{F}}{F}=\frac{\dot{L}}{L}(1-\gamma-m)+\frac{\ddot{L}}{\dot{L}} m
$$

Se puede comprobar fácilmente que:

$$
\frac{\partial \ln \dot{A}}{\partial \ln A}=\frac{(\gamma-(1-m))}{m}
$$

A una velocidad del bastidor de la máquina constante, $(\ddot{L}=0)$, se puede mostrar a partir de la ecuación (28.I) que:

$$
\left.\frac{L}{F}\left(\frac{\partial F}{\partial L}\right)_{i}=\gamma-(1+m)\right)
$$

y, entonces, la carga máxima se alcanza cuando $\gamma=1+$ m. A una velocidad de deformación constante $\left(\frac{\dot{L}}{L}=\frac{\dot{L}}{\dot{L}}=\right.$ Cte. $)$ la carga máxima se alcanza cuando $\gamma=1$, que es el criterio de Considère expresado en la ecuación (18.I). Por otra parte, la condición para el inicio de la localización de la fluencia, es dada en la ecuación (29.I) como $\gamma=1-m$, y está representada en la figura 1 por $\varepsilon_{\text {Hart }}$.

En el caso de un ensayo de compresión, el análisis de Hart es el mismo, cambiando sólo el signo de algunos parámetros. Por ello, $F, \sigma, \varepsilon, \dot{\varepsilon}$ y $\dot{L}$ son negativos; $m, A, \dot{A} y L$ son positivos. Para materiales que sufren un ablandamiento por fluencia, $\delta \sigma / \delta \varepsilon$ es, primero, positivo y, luego, se hace negativo, mientras que $F$ y $\gamma$ son, primero, negativos y, luego, son positivos. Con esta convención de signos, las ecuaciones de estabilidad en el caso de un ensayo de compresión son idénticas que las desarrolladas por Hart en el caso de un ensayo a tracción, excepto que las desigualdades asociadas con la fluencia estable e inestable se invertirían. De esta manera, para $\ddot{L}=0$, la fluencia es estable mientras $(\gamma-(1+m))<0$, e inestable cuando $(\gamma-(1+m))>0$.

Una análisis similar puede aplicarse en el caso de un ensayo a velocidad de deformación verdadera constante (la deformación es estable cuando $((\gamma-1)$ $<0$ ) y en el caso de una fluencia localizada (la fluencia es estable cuando $(\gamma-(1-m)<0)$.

Para ilustrar gráficamente estas relaciones, las deformaciones asociadas con el inicio $\left(\varepsilon_{H}\right)$ y fin $\left(\varepsilon_{H}^{\prime}\right)$ de la fluencia localizada se indican en la figura $3 \mathrm{~b}$ ). Es importante observar que en compresión, $\varepsilon_{H}$ precede a $\varepsilon_{C}$, mientras que el contrario se observa en tracción.

\subsubsection{Criterio de la velocidad de deformación para la localización de la fluencia. Parámetro de la localización de la fluencia}

Poco después de publicar Hart su teoría sobre el ensayo de tracción, Campbell[ ${ }^{[28]}$ presentó su teoría de inestabilidad plástica en materiales sensibles a la velocidad de deformación, estructurando su análisis en términos de un gradiente de deformación axial. Posteriormente, varios autores han utilizado los análisis de Hart y Campbell como puntos de partida para tratar el problema de la inestabilidad plástica, introduciendo una ecuación constitutiva más gene$\mathrm{ral}^{[16,26,27,33 \text { y 34] }}$.

En el análisis de Hart, la estabilidad plástica queda definida en términos de los parámetros de endurecimiento, $\gamma$, y de sensibilidad a la velocidad de deformación, $m$, y la inestabilidad plástica se define como una violación al criterio de estabilidad. No obstante, Hart reconoció que la inestabilidad no tiene por qué ser catastrófica, pero puede proceder a localizar la deformación plástica a un ritmo muy lento. De esta manera, cuando se produce una inestabilidad plástica, el proceso de localización de fluencia controla el grado de deformación útil que se puede aplicar antes de llegar a la rotura final.

Durante el ensayo de tracción de materiales endurecidos por deformación, el parámetro $\gamma$ es, generalmente, positivo y disminuye cuando la deformación aumenta. Por tanto, para materiales con alta sensibilidad a la velocidad de deformación, la deformación se hace inestable a valores bajos de deformación, pero la localización de la deformación no puede producirse hasta que se alcancen altos valores de la deformación. En ensayos de compresión, el conformado de materiales endurecidos por deformaciones es intrínsecamente estable debido a que la tensión de fluencia y el área deformada van aumentando a medida que va progresando la deformación plástica. Sin embargo, para materiales que sufren ablandamiento por fluencia, si la tensión disminuye a una velocidad más grande que la velocidad de incremento del área deformada, la deformación se vuelve inestable. Esto es, la deformación ocurre sin un aumento de la carga aplicada. Jonas, Holt y Coleman ${ }^{[26]}$ han demostrado que la deformación inestable se produce en una zona delimitada por dos valores de deformación: cuando $\gamma=1$, para una velocidad de deformación constante y cuando $\gamma=(1-m)$, para una velocidad del bastidor de la máquina constante. Y, han definido dos tipos de localización: localización de la fluencia (cuando el grado de cambio del área en una zona de inhomogeneidad supera el del volumen de todo el material) y la localización de la velocidad de 
deformación (cuando la velocidad de deformación en una zona de inhomogeneidad supera el del volumen de todo el material). El primer tipo de localización es el mismo definido por Hart y empieza cuando $\gamma=(1-m)$. La localización de la velocidad de deformación es similar a la considerada por Considère y se produce entre los valores de deformación definidos cuando $\gamma=1$ (Fig. 3 b)). Como la sensibilidad a la velocidad de deformación aumenta, los dos valores de deformación correspondientes a $\gamma=(1-m)$ quedan cada vez más alejados y la localización de la fluencia se produce durante un tiempo más largo.

Jonas y Semiatin ${ }^{[16,26,27 \text { y 33] }}$ trataron de definir un parámetro capaz de cuantificar el grado de la localización de la fluencia. Empezaron sus análisis, de la misma manera que procedió Hart, considerando que la carga, $F$, es igual en todos los puntos de la pieza y aplicando una variante de la ecuación (10.I):

$$
\partial F=0=\sigma \partial A+A \partial \sigma=1+\frac{A}{\sigma}\left(\frac{\partial \sigma}{\partial A}\right)_{F}
$$

sabiendo que:

$$
\partial \sigma=\left(\frac{\partial \sigma}{\partial \varepsilon}\right) \partial \varepsilon+\left(\frac{\partial \sigma}{\partial \dot{\varepsilon}}\right) \partial \dot{\varepsilon}
$$

la ecuación (31.I) puede volverse a escribir de la siguiente manera:

$$
\frac{A}{\sigma} \frac{\left(\frac{\partial \sigma}{\partial \varepsilon}\right) \partial \varepsilon+\left(\frac{\partial \sigma}{\partial \dot{\varepsilon}}\right) \partial \dot{\varepsilon}}{\partial A}=-1
$$

En la ecuación (33.I) puede usarse la definición de los parámetros $\gamma, m$ y $\partial \varepsilon=-\frac{\partial A}{A}$ de modo que resulta:

$$
\left.\gamma-m \frac{A}{\dot{\varepsilon}} \frac{\partial \dot{\varepsilon}}{\partial A}\right)_{P}=1
$$

o bien,

$$
\left.\frac{A}{\dot{\varepsilon}} \frac{\partial \dot{\varepsilon}}{\partial A}\right)_{P}=\frac{\gamma-1}{m}=\alpha
$$

El parámetro $\alpha$ puede definirse, también, como, $\alpha=\frac{-\partial \ln \dot{\varepsilon}}{\partial \varepsilon}$ y se usa para estimar la tendencia que tiene el material a desarrollar zonas de concentración de la deformación. Es interesante destacar que $\alpha$ es función, solamente, de los parámetros característicos del material $\gamma$ y $m$. Ambos o cada uno de estos parámetros pueden ser función de $\varepsilon, \dot{\varepsilon}$ y $T$. Así, para determinar la tendencia del material a desarrollar concentraciones de deformación, se requieren unas curvas de fluencia obtenidas durante ensayos de compresión uniaxial, a velocidad de deformación verdadera constante y valores estimados de $m$ (basados en las curvas de fluencia para dos velocidades de deformación diferentes), y $\alpha$ debe ser evaluado en función de la deformación, velocidad de deformación y temperatura.

La ecuación (35.I) indica que la fluencia es estable en un ensayo de tracción cuando $\alpha>0$ y es estable en un ensayo de compresión cuando más ne-

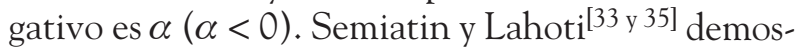
traron que la tensión de fluencia máxima es una condición necesaria para la aparición de la localización de la deformación y de las bandas de cizalladura, pero no es suficiente y debe haber un grado sustancial de ablandamiento por fluencia (endurecimiento negativo) para que pueda haber una localización considerable de la deformación. Dichos autores, siguiendo la metodología desarrollada por Jonas et al. [26 y 27] correlacionaron la aparición de la localización de la fluencia con las propiedades del material, a través del parámetro de localización de la fluencia $\alpha$. En ensayos de compresión, la fluencia localizada se produce cuando $\alpha>0$; esto corresponde a materiales que exhiben coeficientes de endurecimiento, $\gamma>1$, es decir, un ablandamiento por fluencia bastante importante (como $\sigma$ y $\varepsilon$ son, ambas, negativas en compresión). Cuando $\gamma=1$, la velocidad de ablandamiento por fluencia solo se compensa por el endurecimiento geométrico o por incremento de área. Así, $\gamma>1$ es necesaria para permitir el desarrollo de una fluencia inestable. Generalmente, los materiales que se endurecen por deformación, o bien los que presentan un ablandamiento por fluencia mínimo, resisten apreciablemente las concentraciones de deformación (esto es, tienen muy bajas velocidades de localización de la fluencia) siempre y cuando la sensibilidad a la velocidad de deformación sea suficientemente alta $(\geq 0,2)$.

Varios trabajos de investigación ${ }^{[16,33}$ y 36], en base a observaciones microestructurales, verificaron que, en procesos de conformado en caliente, las aleaciones de titanio exhiben una localización de la fluencia significativa cuando $\alpha \geq 5$. Así, el criterio de inestabilidad plástica, basado en conceptos de localización de la

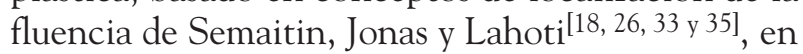


procesos de conformado en caliente puede expresarse de la siguiente manera:

$$
1-\frac{\alpha}{5}<0
$$

\subsection{Criterios termodinámicos continuos}

En las últimas décadas, varios investigadores han estudiado extensivamente los efectos de la deformación, velocidad de deformación, temperatura y microestructura sobre el comportamiento de fluencia de los metales utilizando mapas de procesado $(\text { Raj })^{[3]} \mathrm{O}$ de deformación (Ashby y Frost ${ }^{[4]}$. En estas dos aproximaciones, las ecuaciones constitutivas utilizadas se desarrollaron asumiendo que son dependientes de procesos atómicos básicos, tales como movimiento de dislocaciones, difusión, deslizamiento de los límites de grano y transformaciones de fase. Tanto los mapas de Ashby y Frost como los de Raj constituyeron un avance muy importante en la optimización de la conformabilidad, puesto que proporcionan información de las zonas de inestabilidades plásticas a evitar durante un proceso de conformado. Sin embargo, dichos mapas presentan ciertas limitaciones para aleaciones comerciales debido a la no disponibilidad de todos los datos requeridos para su construcción. Además, no es posible saber, a priori, todos los mecanismos microestructurales de daño que ocurren en el material para poder modelarlos adecuadamente.

Debido a las limitaciones de los mapas de deformación y de procesado, se desarrolló otro método muy prometedor en cuanto al análisis de la inestabilidad plástica y la optimización de la conformabilidad en caliente; es el llamado "Modelo Dinámico de Materiales" (de aquí en adelante, DMM). En este método, desarrollado por Prasad et al..$^{[5}$ y 7$]$ y analizado por Gegel et al. ${ }^{[37 \text { y 38] y Alexander }}{ }^{[39]}$, la pieza conformada a alta temperatura se considera como la única parte de todo el sistema del proceso capaz de disipar energía y su ecuación constitutiva es una relación analítica que describe la variación de la tensión de fluencia con los parámetros de deformación, es decir, temperatura y velocidad de deformación. Esta ecuación es una característica intrínseca del material de la pieza conformada y describe la manera en que la energía se convierte en cualquier instante, usualmente, en energía térmica y microestructural no recuperable por el material. Por consiguiente, la deformación en caliente se modela manejando varios procesos termodinámicos irreversibles controlados por la velocidad de energía aportada y la posterior disipación de esta energía mediante los procesos metalúrgicos dinámicos.
El desarrollo del DMM se ha basado en tres grandes disciplinas: 1: Mecánica continua de la fluencia plástica en grandes deformaciones; donde el material conformado actúa como un disipador de energía, tal como ha sido descrito por Ziegler ${ }^{[40]}$; 2: Principios generales de la modelización de sistemas físicos descritos por Wellstead ${ }^{[41]}$, donde los conceptos energéticos de contenido disipador y co-contenido disipador se aplican a sistemas mecánicos, eléctricos y magnéticos; 3: Conceptos de la termodinámica irreversible que describen la estabilidad y la autoorganización de los sistemas caóticos propuestos por Prigogene ${ }^{[42]}$, donde la tasa de producción de entropía se utiliza para caracterizar el comportamiento de los fenómenos irreversibles.

A pesar de ser validado por numerosos trabajos de investigación ${ }^{[9,13}$ y 43-52], el DMM sigue siendo un tema de controversia en la literatura y no se puede afirmar que es un método "de consenso", debido a las inconsistencias que varios investigadores encontraron en sus conceptos y fundamentos físicos que explicaremos más adelante en este trabajo. No obstante, las comprobaciones microestructurales, llevadas a cabo en diferentes procesos industriales (forja, extrusión y laminación) de varios materiales ${ }^{[9]}$, avalan la utilidad de este método en la identificación de los dominios de inestabilidades plásticas en los mapas de conformado.

\subsubsection{Conceptos termodinámicos}

En los modelos termodinámicos, el conformado en caliente se analiza por medio de la energía suministrada, $P$, al material conformado. La energía total disipada está relacionada con la tasa de producción de entropía de la siguiente manera:

$$
P=\sigma \cdot \dot{\varepsilon}=\frac{d S}{d t} T \geq 0
$$

donde, $\frac{d S}{d t}$ es la tasa de producción de entropía. La energía, $P$, es siempre positiva para los procesos irreversibles (deformación plástica, por ejemplo) e igual a cero para procesos reversibles. Bajo condiciones isotérmicas, la tasa de producción de entropía es totalmente "interna" $(P)$. El teorema de la mínima producción de entropía de Prigogine ${ }^{[53]}$ postula que todo sistema evoluciona naturalmente a un estado estacionario estable donde la tasa de producción de entropía es mínima; este estado corresponde al de máxima eficiencia en presencia de procesos irre versibles. En el marco de la teoría termodinámica 
generalizada de los procesos irreversibles se puede postular que todo sistema evoluciona, en la superficie definida por la producción global de energía y los parámetros de control, a un estado donde la disipación termodinámica es mínima. Una de las aplicaciones potenciales de la termodinámica de los procesos irreversibles consiste en establecer las condiciones iniciales y de frontera bajo las cuales un sistema logra obtener el máximo de eficiencia con un mínimo de disipación termodinámica. Basaran y Nie ${ }^{[54]}$ han demostrado que la producción de entropía causada por la disipación de energía puede dividirse en cuatro términos: dos, llamados disipación intrínseca ó disipación mecánica, que consisten en una disipación plástica más una disipación asociada a la evolución de otras variables internas y los otros dos términos, que son la disipación térmica debida a la conducción de calor y una fuente interna de calor.

En los casos donde los cambios microestructurales y la disipación de calor pueden separarse, Malvern ${ }^{[55]}$, también, ha demostrado que la producción total de entropía consiste en dos partes complementarias. La primera parte (generalmente, más grande) consiste en "entropía de conducción" que se debe a la conducción de calor desde donde se genera (por fluencia plástica) hacia las zonas más frías del sistema. La segunda parte se debe a la disipación microestructural, que provoca una reducción en la tensión de la fluencia plástica (por movimiento de dislocaciones). Ziegler ${ }^{[40]}$ representó estas dos partes en términos de funciones disipativas y demostró que la energía instantánea total disipada $(\mathrm{P}=\sigma \cdot \dot{\varepsilon})$ puede expresarse por la suma de dos integrales ${ }^{[41]}$ :

$$
P=\sigma \cdot \dot{\varepsilon}=\int_{0}^{\dot{\varepsilon}} \sigma \cdot d \dot{\varepsilon}+\int_{0}^{\sigma} \dot{\varepsilon} \cdot d \sigma=G+J
$$

donde, $G$ es el área por debajo de la curva de la figura 4 a) y representa el contenido disipador. J es el área por encima de la curva de la figura 4 b) y es la función complementaria de $\mathrm{G}$.

Para entender el sentido físico de $G$ y J, deben considerarse los procesos microscópicos de la deformación plástica ${ }^{[40]}$. La fluencia plástica ocurre por deslizamiento cristalográfico que, como se sabe, resulta del desplazamiento de dislocaciones sobre sus planos de deslizamiento bajo la acción de una tensión de cizalladura, $\tau$. La acción de $\tau$ aumenta la energía cinética y potencial. Una parte considerable de la energía potencial es, casi instantáneamente, transformada en energía cinética. Sin embargo, la energía cinética total producida por fluencia plástica se transforma en calor. La mayor porción de esta transformación se disipa a través de este aumento de temperatura y se representa por el contenido disipador $G$ (el área bajo la curva, como indica la figura 4) y la parte restante es almacenada en forma de defectos.

Las dislocaciones móviles se agrupan, después de la aniquilación de algunas por la restauración térmica

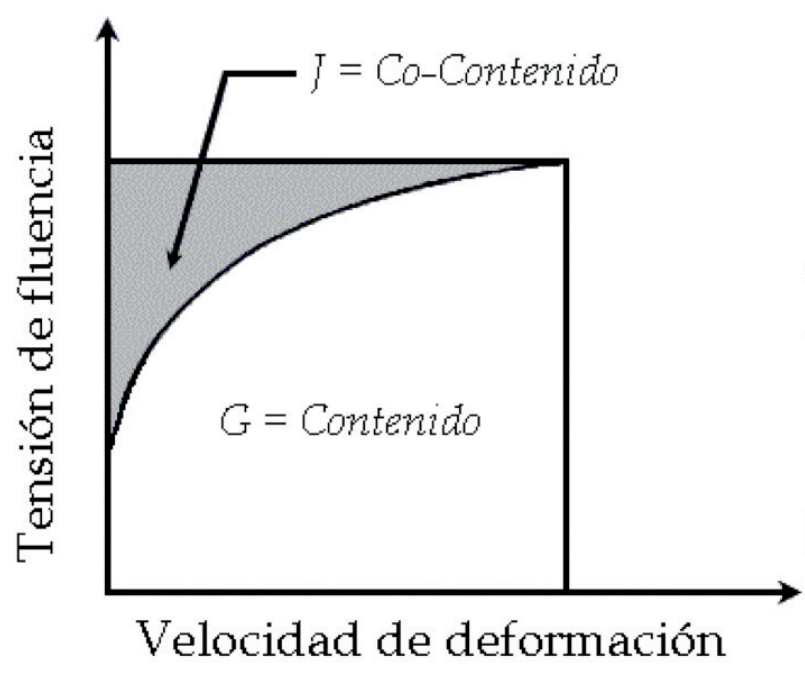

(a)

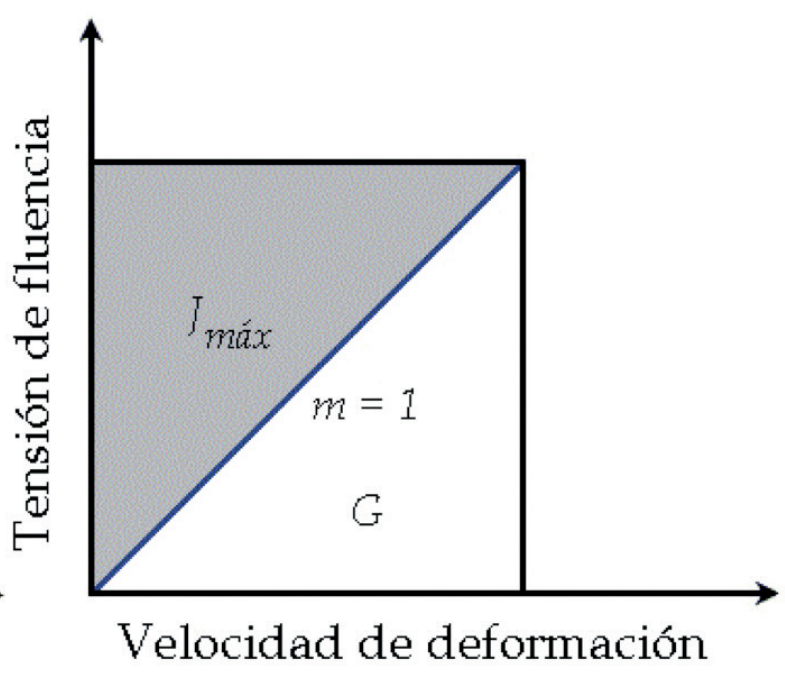

(b)

Figura 4. a) Representación esquemática de la ecuación constitutiva de un disipador de energía mostrando las áreas correspondientes a las funciones $G$ y J; b) Caso de un disipador lineal ideal

Figure 4. a) Schematic representation of the dynamic constitutive equation in a power dissipator showing the areas corresponding to the functions $\mathrm{G}$ and $\mathrm{J}$; b) Case of an ideal linear dissipator. 
o mecánica y pueden, también, formar intercaras que a temperaturas suficientemente altas pueden migrar para causar una aniquilación, a mayor escala, de las dislocaciones. A temperaturas relativamente bajas, donde los procesos de restauración son lentos, los grupos de dislocaciones pueden crear fisuras internas, las superficies libres de las cuales forman "los sumideros" para la aniquilación de las dislocaciones. Pueden existir otros procesos microscópicos (p.ej., fluencia difusional, transformación de fase inducida por tensión) que eliminan dislocaciones y disipan energía. Todos estos fenómenos metalúrgicos contribuyen a la disipación de energía en menor proporción que el contenido disipador $\mathrm{G}$ y representan la disipación de energía a través de la función complementaria J: el co-contenido disipador. El comportamiento dinámico del material puede, explícitamente, modelarse en términos de la variación de $J$ con los parámetros del proceso. En cada instante, la disipación de energía ocurre mediante un aumento de temperatura (el contenido, $G$ ) y cambios microestructurales (el co-contenido, $J$ ).

El reparto de energía entre $G$ y $J$ se decide mediante el coeficiente $m$ de sensibilidad a la velocidad de deformación de la tensión de fluencia. De la ecuación (38.I) se deduce que el reparto de energía entre $G$ y $J$ viene dada por:

$$
\left(\frac{\partial J}{\partial G}\right)_{\varepsilon, T}=\frac{\partial P}{\partial G} \frac{\partial J}{\partial P}=\frac{\sigma d \dot{\varepsilon}}{\dot{\varepsilon} d \sigma}=\left[\frac{\partial(\ln \sigma)}{\partial(\ln \dot{\varepsilon})}\right]_{\varepsilon, T} \equiv m
$$

para una fluencia plástica estable, $0<m<1$, es decir, la deformación ocurre entre estos dos extremos $J=0$ y $J=0,5$ P. En el caso extremo, $J$ puede ser como mucho, igual a $G$, puesto que las dislocaciones no pueden ser aniquiladas a velocidades más grandes que aquéllas a las que se generan. Esto es el caso ideal de un disipador lineal, donde $m=1$ y $J=J_{\max }=G_{\text {min }}=0,5 P$ y la mitad de energía se disipa como fluencia plástica del material mientras que la otra mitad se disipa en forma de calor (Fig. 4 b). En el otro extremo, $m=0$ y $J=0$ la deformación es totalmente independiente de la velocidad de deformación y el material no disipa energía a través de los procesos metalúrgicos y se comportara como un "almacén" de energía mediante generación de dislocaciones.

En materiales que tienen microestructuras complejas o aleaciones bifásicas, los procesos metalúrgicos dinámicos, que contribuyen a la disipación de energía durante la deformación en caliente, ocurren simultáneamente y/o interactivamente. En consecuencia, el valor evaluado de J será el resultado global de estas interacciones. En efecto, los procesos metalúrgicos, tales como la restauración dinámica, recristalización dinámica, rotura interna, disolución o crecimiento de partículas (o segundas fases) en condiciones dinámicas y transformación de fase inducida por deformación o precipitación dinámica contribuyen a un cambio en el valor de la energía disipada, J. Cuando dos procesos principales de disipación de características diferentes ocurren simultáneamente, el valor de $J$ alcanza su máximo cuando la energía disipada por uno iguala la del otro.

Considerando que la velocidad máxima posible de aniquilación de dislocaciones solo puede ser, como mucho, igual a la velocidad de generación de estas dislocaciones, la disipación de energía a través del co-contenido, $J$, puede normalizarse con un disipador lineal $\left[m=1 ; J_{\max }=\left(\frac{P}{2}\right)\right]$ para definir un parámetro adimensional llamado eficiencia de la disipación de energía $(\eta)$ a través de los procesos metalúrgicos (Ec. (5.I)).

\subsubsection{Criterios de estabilidad de Liapunov}

El parámetro adimensional de la eficiencia de disipación de energía, $\eta=\frac{2 m}{m+1}$, del DMM, es muy importante para el control de la energía disipada du-

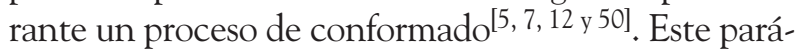
metro se utiliza, también, para formular la función de Liapunov, que es una cantidad del sistema asociada al criterio de estabilidad de Liapunov ${ }^{[56]}$ considerado como un método general aceptado en el diseño ingenieril[57]. La función de Liapunov es un término arbitrario que relaciona los cambios en la energía total de un sistema dado.

El criterio de Liapunov requiere que el sistema minimice su energía total, continuamente. Además, este criterio conduce a la condición de que, en regiones estables, la derivada parcial de segundo orden de los parámetros de eficiencia, $\eta$, y de entropía, $S$, respecto a $\log \dot{\varepsilon}$ tiene que ser negativa. Esta condición asegura, definitivamente, que el sistema se aproxime a las condiciones del estado estable donde la energía es mínima y la eficiencia es máxima. La función de Liapunov se formula de la manera siguiente:

$$
V_{1}=\eta(\log \dot{\varepsilon})
$$

Para definir un parámetro de control capaz de estudiar la influencia de la temperatura sobre el comportamiento y la conformabilidad del material se ha establecido una metodología, basada en el método directo de Liapunov, donde la energía total disipada se representará en términos de la velocidad de entropía aplicada, $S_{\text {app }}$ y de la temperatura. Dicha metodología se ha utilizado para determinar la relación 
entre la velocidad de entropía producida por el sistema, $S_{s y s,}$, la velocidad de entropía aplicada al sistema, $S_{a p p}$, usando las siguientes etapas:

$$
P=\sigma \dot{\varepsilon}=S_{a p p} T
$$

y por definición,

$$
\left.\frac{\partial P}{\partial T}\right|_{\dot{s}}=\dot{S}_{s y s}
$$

además,

$$
\frac{\partial P}{\partial T}=-\frac{P}{T^{2}} \frac{\partial \ln P}{\partial(1 / T)}=\frac{P}{T}\left[-\frac{1}{T} \frac{\partial(\ln P)}{\partial(1 / T)}\right]
$$

donde,

$$
\left.\frac{1}{T} \frac{\partial(\ln P)}{\partial(1 / T)}\right|_{\dot{\varepsilon}}=-\frac{1}{T} \frac{\partial(\ln \sigma)}{(1 / T)}
$$

define un nuevo parámetro de control, $s$, llamado parámetro de sensibilidad a la temperatura, definido como

$$
s=\left[-\frac{1}{T} \frac{\partial(\ln \sigma)}{\partial(1 / T)}\right]_{\varepsilon, \dot{\varepsilon}}
$$

El signo menos en la ecuación (45.I) puede ser despreciado y el valor de $s$ puede considerarse como positivo porque la tensión de fluencia disminuye cuando la temperatura aumenta. Por tanto, la velocidad de entropía producida por el sistema viene dada por:

$$
\dot{S}_{s y s}=\frac{P}{T} S
$$

donde, $\mathrm{P} / \mathrm{T}$ puede considerarse como la velocidad de entropía aplicada al sistema.

El parámetro de sensibilidad a la temperatura, $s$, puede ser calculado usando los valores experimentales de $s$ obtenidos para una deformación y una velocidad de deformación constante a intervalos muy próximos de temperatura.

$$
\mathrm{s}=\left.\frac{\partial \ln \sigma}{T \partial(1 / T)}\right|_{\varepsilon, \dot{\varepsilon}}=\frac{\ln \sigma_{2}-\ln \sigma_{1}}{\left(\frac{T_{1}+T_{2}}{2}\right)\left[\frac{T_{1}-T_{2}}{T_{1} T_{2}}\right]}
$$

Este valor de $s$ corresponderá a la temperatura:

$$
T=\frac{T_{1}+T_{2}}{2}
$$

De la misma manera, $s$ se determina para varias temperaturas y velocidades de deformación a una deformación dada, y estos valores serán, posteriormente, usados para la determinación de $\frac{\partial s}{\partial \ln \dot{\varepsilon}}$.

De acuerdo con la $2^{2}$ ley de la termodinámica, $s$ debería ser más grande que la unidad para una fluencia estable del material. Esto quiere decir que la pieza conformada debería almacenar entropía al menos tan rápido como la velocidad de producción de calor por deformación para la fluencia estable. Por lo tanto, para una fluencia estable, $\frac{\partial s}{\partial \ln \dot{\varepsilon}}$ tiene que ser inferior a 0 , si s es considerada como una función de Liapunov, es decir:

$$
V_{2}=s(\ln \dot{\varepsilon})
$$

Por tanto, la condición $\frac{\partial \sigma}{\partial T}<0$ es muy importante y sirve para delinear las zonas de estabilidad en los mapas de disipación de energía. Dicha condición asegura que el sistema se aproxime a un estado de mínima energía y máxima eficiencia.

\subsubsection{Criterios de estabilidad de Gegel}

Usando los fundamentos de la mecánica de medios continuos, de la termodinámica y de la teoría de estabilidad, Gegel ${ }^{[37}$ y 38$]$ ha sugerido para una fluencia estable, durante un proceso de conformado, las siguientes condiciones considerando una función de Liapunov, $L(\eta, s)^{[58]}$ :

$$
0<m \leq 1
$$

$$
\frac{\partial \eta}{\partial \ln \dot{\varepsilon}}<0
$$

$$
s \geq 1
$$

$$
\frac{\partial s}{\partial \ln \dot{\varepsilon}}<0
$$


Desde un punto de vista metalúrgico y para una fluencia estable (Ec. (50.I)), los valores del parámetro $m$ pueden deducirse de las consideraciones teóricas y de las observaciones

$$
\begin{aligned}
G<P & \Rightarrow \frac{d G}{d \dot{\varepsilon}}<\frac{d P}{d \dot{\varepsilon}} \Rightarrow \sigma<\dot{\varepsilon} \frac{\partial \sigma}{\partial \dot{\varepsilon}}+\sigma \\
& \Rightarrow 1<\frac{\dot{\varepsilon}}{\sigma} \frac{\partial \sigma}{\partial \dot{\varepsilon}}+1 \Rightarrow 1<m+1 \\
& \Rightarrow m>0
\end{aligned}
$$

Para una fluencia plástica estable la evolución de la tensión, $\sigma$, con $\dot{\varepsilon}$ tiene que ser convexa y para una fluencia plástica ideal, $G=J$, por tanto, se puede escribir:

$$
\begin{gathered}
J \leq G \\
\frac{d J}{d G} \leq 1 \Rightarrow m \leq 1
\end{gathered}
$$

La ecuación (50.I) satisface las relaciones dadas en las ecuaciones (54.I) y (56.I).

Usando la ecuación (45.I) la condición de estabilidad (52.I) puede expresarse en términos de tensión de fluencia, de la siguiente manera:

$$
-\frac{\partial \sigma}{\partial T} \geq \frac{\sigma}{T}
$$

lo que significa que $\frac{\partial \sigma}{\partial T}<0$. Lo cual indica que la tensión de fluencia, $\sigma$, disminuye con la temperatura, $T$, a cualquier deformación y velocidad de deformación. Y, a partir de la ecuación (50.I), la condición de estabilidad (53.I) puede expresarse como:

$$
\begin{aligned}
\frac{1}{T} \frac{\partial}{\partial(1 / T)}\left(\frac{\partial \ln \sigma}{\partial \ln \dot{\varepsilon}}\right)<0 & \Rightarrow \frac{1}{T} \frac{\partial m}{\partial(1 / T)}<0 \\
& \Rightarrow-T \frac{\partial m}{\partial T}<0 \\
& \Rightarrow \frac{\partial m}{\partial T}>0
\end{aligned}
$$

lo que indica que, para una fluencia estable, la sensibilidad a la velocidad de deformación debe aumentar con la temperatura.

De esta manera, de los análisis anteriores se puede concluir que para que la fluencia plástica de un material sea estable, además de satisfacer las dos condiciones, $0<m<1$ y $\frac{\partial m}{\partial T}>0$, la curva de evolución de $\sigma$ con $\dot{\varepsilon}$ debe ser convexa y la tensión de fluencia debe disminuir con la temperatura.

\subsubsection{Criterios de estabilidad de Alexander}

Usando el criterio de estabilidad de Liapunov y considerando una función $L(m, s)$, Alexander ${ }^{[39]}$ ha propuesto las siguientes condiciones para una fluencia plástica estable durante un proceso de conformado:

$$
\begin{gathered}
0<m \leq 1 \\
\frac{\partial m}{\partial \ln \dot{\varepsilon}}<0 \\
s \geq 1 \\
\frac{\partial s}{\partial \ln \dot{\varepsilon}}<0
\end{gathered}
$$

Las condiciones anteriores indican que, para una fluencia estable, la curva de evolución de $\sigma$ con $\dot{\varepsilon}$ debe ser convexa, la tensión de fluencia debe disminuir con la temperatura y $m$ debe disminuir con la velocidad de deformación y aumentar con la temperatura.

\subsubsection{Criterio de inestabilidad basado en la teoría de fluencia plástica de Ziegler}

Algunos principios extremos en la termodinámica irreversible aplicada a la mecánica continua de la fluencia plástica en las grandes deformaciones han sido considerados por Ziegler ${ }^{[40]}$. La producción de entropía puede depender del estado del sistema y de su historia, determinada por el incremento de deformación $\left(d x_{k}\right)$. Además, puede expresarse en términos de la temperatura $(T)$ y del trabajo de disipación elemental $\left(d W^{(i)}\right)$.

$$
\mathrm{dW}^{(\mathrm{i})}=\mathrm{X}_{k}^{(\mathrm{i})} \cdot \mathrm{dx} \mathrm{k}_{\mathrm{k}}=T \cdot \mathrm{d}^{(\mathrm{i})} \mathrm{S} \geq 0
$$

donde, $X_{k}^{(i)}$ es la fuerza irreversible.

La velocidad del trabajo de disipación está relacionada con la tasa de producción de entropía, de la manera siguiente:

$$
\frac{\mathrm{dW}^{(1)}}{\mathrm{dt}}=\mathrm{P}^{(\mathrm{i})}=\mathrm{X}_{\mathrm{k}}^{(\mathrm{i})} \cdot \dot{\mathrm{x}}_{\mathrm{k}}=\frac{T \cdot \mathrm{d}^{(\mathrm{l})} \mathrm{S}}{\mathrm{dt}} \geq 0
$$


donde, $\dot{x}_{k}$ es la velocidad de deformación y $P^{(i)}$ la energía disipada.

En un estado dado del proceso, la velocidad del trabajo de disipación es una función de disipación, $D\left(\dot{x}_{k}\right)$, del sistema.

$$
\mathrm{P}^{(\mathrm{i})}=\mathrm{D}\left(\dot{\mathrm{X}}_{\mathrm{k}}\right)
$$

La función de disipación representa el comportamiento constitutivo del material y se define por:

$$
\mathrm{D}\left(\dot{\mathrm{x}}_{\mathrm{k}}\right)=T \frac{\mathrm{d}^{(\mathrm{i})} \mathrm{S}}{\mathrm{dt}} \geq 0
$$

La fuerza irreversible, $X_{k}^{(i)}$, está relacionada con $D\left(\dot{x}_{k}\right)$ mediante la expresión:

$$
\mathrm{X}_{\mathrm{k}}^{(\mathrm{i})} \dot{\mathrm{X}}_{\mathrm{k}}=\mathrm{D}\left(\dot{\mathrm{X}}_{\mathrm{k}}\right) \geq 0
$$

Sobre la base de la función de disipación y el principio de la fuerza irreversible mínima Ziegler demostró que la fluencia estable ocurre si el cociente diferencial cumple la desigualdad:

$$
\left.\frac{\partial D}{\partial R}\right\rangle \frac{D}{R}
$$

donde, $R=\left(\dot{x}_{k} \cdot \dot{x}_{k}\right)^{1 / 2}$. Esto es equivalente a decir que la fuerza irreversible, $X_{k}^{(i)}$, aumentará con la velocidad $\dot{x}_{k}$.

Prasad $^{[7]}$ y Kumar ${ }^{[59]}$ desarrollaron un criterio para predecir los dominios de inestabilidades de fluencia durante el conformado de un material. Este criterio se basa en los principios de la mecánica de los medios continuos aplicados a procesos de fluencia plástica en grandes deformaciones propuestos por Ziegler. Puesto que el co-contenido disipador, J, es responsable de la disipación de energía mediante procesos metalúrgicos, la función de disipación relacionada con la estabilidad de fluencia está representada por J. Reemplazando D por J en la ecuación (68.I) se puede obtener la condición para una fluencia estable a una deformación y temperatura constante:

$$
\frac{\partial J}{\partial \dot{\varepsilon}}>\frac{J}{\dot{\varepsilon}}
$$

La ecuación (69.I) puede escribirse, también, de la siguiente manera:

$$
\frac{\partial \ln J}{\partial \ln \dot{\varepsilon}}>1
$$

Asumiendo que la ecuación dinámica constitutiva del material conformado sigue una ley potencial, $\sigma=K \cdot \dot{\varepsilon}^{m}$, obtuvieron que el co-contenido disipador $J$, puede expresarse como:

$$
J=\int_{0}^{\sigma} \dot{\varepsilon} \cdot d \sigma=\frac{m}{m+1} \sigma \dot{\varepsilon}=\frac{m P}{m+1}
$$

Tomando el logaritmo natural en ambos lados de la ecuación (71.I):

$$
\ln J=\ln \left(\frac{m}{m+1}\right)+\ln \sigma+\ln \dot{\varepsilon}
$$

y diferenciando la ecuación (72.I) con respecto a se obtiene:

$$
\frac{\ln J}{\ln \dot{\varepsilon}}=\frac{\partial\left(\ln \left(\frac{m}{m+1}\right)\right)}{\partial(\ln \dot{\varepsilon})}+\frac{\partial \ln \sigma}{\partial \ln \dot{\varepsilon}}+1
$$

Sustituyendo la ecuación (71.I) en la ecuación (70.I) y sabiendo que $m=\left(\frac{\partial \ln \sigma}{\partial \ln \dot{\varepsilon}}\right)=\frac{\dot{\varepsilon}}{\sigma}\left(\frac{\partial \sigma}{\partial \dot{\varepsilon}}\right)_{\varepsilon}$, se obtiene la condición de estabilidad:

$$
\xi(\dot{\varepsilon})=\frac{\partial / \mathrm{n}(\mathrm{m} / \mathrm{m}+1)}{\partial / \mathrm{n} \dot{\varepsilon}}+m>0
$$

Por lo tanto, la $\xi(\dot{\varepsilon})$ variación de con la temperatura y la velocidad de deformación constituye el mapa de inestabilidad que puede ser superpuesto sobre el mapa de disipación de energía. El mapa de inestabilidad delinea las regiones de inestabilidad donde el parámetro $\xi(\dot{\varepsilon})$ es negativo y serán, por tanto, zonas a evitar en el proceso de conformado.

\subsubsection{Criterio de inestabilidad plástica basado en el modelo de reciprocidad polar}

El modelo de reciprocidad polar (PRM) ${ }^{[60 \text { y } 61]}$ se basa en el principio de fluencia de la teoría de plasticidad, que a su vez está basada sobre la convexidad 
de las superficies potenciales ${ }^{[62]}$. Este modelo se ha concebido para expresar la energía en dos términos complementarios; el de energía de endurecimiento, $\dot{P}_{E}=\int_{0}^{\dot{\varepsilon}} \sigma d \dot{\varepsilon}$, y el de energía de disipación, $\dot{P}_{D}=\int_{0}^{\sigma} \dot{\varepsilon} d \sigma$. De esta manera, la energía total se puede expresar como:

$$
\sigma \dot{\varepsilon}=\dot{P}_{E}+\dot{P}_{D}=\int_{0}^{\varepsilon} \sigma d \dot{\varepsilon}+\int_{0}^{\sigma} \dot{\varepsilon} d \sigma
$$

Además, en este modelo el parámetro de conformabilidad intrínseca, $\zeta$, viene dado por:

$$
\zeta=\left(\frac{\dot{P}_{E}}{\dot{P}_{E \min }}\right)-1=\frac{2}{\sigma \dot{\varepsilon}} \int_{0}^{\dot{\varepsilon}} \sigma d \dot{\varepsilon}-1=\left(\frac{2 G}{\sigma \dot{\varepsilon}}-1\right)(76 . \mathrm{I})
$$

donde,

$$
\dot{P}_{E \min }=\frac{\sigma \dot{\varepsilon}}{2}
$$

A grandes velocidades de deformación, donde falla el principio de fluencia, el término de energía de endurecimiento, $\dot{P}_{E}$, aumenta monótonamente para alcanzar un valor muy grande para el cual el parámetro $\zeta$ tienda a ser igual a 1 . En estas condiciones, el término de disipación de energía, $\dot{P}_{D}$, alcanza un extremo catastrófico y el material muestra una inestabilidad plástica en su comportamiento en fluencia. En el modelo dinámico de materiales, los términos y representan el contenido disipador, $G$, y el co-contenido disipador, $J$, respectivamente. Usando la expresión del parámetro de la eficiencia de disipación de energía expresada en la ecuación (5.I), la ecuación (76.I) puede volverse a escribir tal como sigue:

$$
\zeta+\eta=1
$$

A partir de ésta última ecuación, Rajagopalachary y Kutumbarao ${ }^{[60 \text { y } 61]}$ establecieron que un valor de $\zeta$ que se aproxime a la unidad es una condición de inestabilidad suficiente y definitiva para determinar los dominios de inestabilidades metalúrgicas en los mapas de $\zeta$ y no se requiere de ningún otro criterio de estabilidad.

Sin embargo, según la relación (78.I), cuando el valor de $\zeta$ tiende a ser igual a la unidad, el valor de $\eta$ tiende a ser igual a cero. Esto significa que $J=0$ y $G$ $=P$. En este caso, toda la energía suministrada al material, durante el proceso de conformado, se disipará en forma de calor. Dicha disipación podría conducir a la aparición de inestabilidades plásticas a través de procesos continuos (p.ej. cizallamiento adiabático).

La condición de inestabilidad cualitativa, $\varsigma \rightarrow 1$, propuesta en el modelo de reciprocidad polar es inadecuada para delinear todos los dominios de fluencia inestable, lo que llevó a pensar en la necesidad de establecer una condición adicional cualitativa capaz de garantizar la estabilidad en una fluencia plástica estable ${ }^{[63]}$, y es la siguiente:

$$
\eta>0 \quad \text { ó } \quad \varsigma<1
$$

\section{EVALUACIÓN CRÍTICA DEL FUNDAMENTO FÍSICO DEL DMM}

\subsection{Análisis crítico de Montheillet}

Montheillet ${ }^{[64]}$ ha examinado el fundamento físico del co-contenido disipador, J, del DMM y lo ha criticado duramente: afirma que la interpretación física de la disipación de energía ni sigue las directrices de la mecánica de los medios continuos ni se basa en leyes que rigen el comportamiento de los materiales, pero se puede considerar como una aproximación heurística basada en la disipación de energía durante el conformado de materiales. De su reflexión, se puede apreciar que la eficiencia de disipación de energía $\eta$, tal como está definida a partir del co-contenido disipador, $J$, tiene un sentido físico muy confuso y sería científicamente correcto establecer un procedimiento similar basado directamente en el parámetro de sensibilidad a la velocidad de deformación, $m$.

Para entender el sentido físico de $m$ y su importancia como parámetro de control de la conformabilidad, se puede hacer la analogía siguiente con la $2^{\underline{a}}$ ley de Newton: para describir la localización de la fluencia se considera una fuerza motriz, $F$, y el coeficiente de sensibilidad, $m$, juega el papel de una "masa", $M$, mientras que la "aceleración", $a$, es la velocidad de localización de la fluencia. De esta manera, en presencia de una fuerza motriz fija, los valores altos de $m$ son los responsables directos de las bajas velocidades de localización de la fluencia. De la discusión anterior, se deduce que la tendencia de la localización de la fluencia, durante el conformado, se reduce al mínimo cuando el rango seleccionado de temperatura y velocidad de deformación es al que corresponden los valores más altos de $m$. Esta interpretación está en acuerdo con las teorías de inestabilidades plásticas basadas en los trabajos de Considère y Hart y, según las cuales, se considera que la localización de la fluencia no puede manifestarse hasta que la velocidad de endurecimiento alcance un valor 
crítico, que depende de las condiciones de contorno. Una vez alcanzado este valor, la cinética de la localización de la fluencia dependerá del coeficiente de sensibilidad a la velocidad de deformación, $m$.

Montheillet concluyó su análisis crítico reclamando que la utilización del parámetro $h$ para la determinación de los dominios estables en los mapas de procesado no puede considerarse apropiado.

En su réplica a Montheillet, Prasad ${ }^{[10,65}$ y 66] expli- $^{-}$ ca la interpretación física de las dos funciones, G y $J$, basada en los principios de la termodinámica, y pretende que el concepto de la maximización de $\eta$ para el análisis de los problemas de los procesos de conformado está sostenido por numerosos trabajos de investigación microestructurales, incluyendo los que confirman la existencia de inestabilidades plásticas, en una amplia gama de materiales. El DMM se desarrolló explorando la naturaleza viscoplástica del conformado de materiales a altas temperaturas e interpretando físicamente el parámetro de sensibilidad, a la velocidad de deformación, como un factor de reparto de energía que permite la evaluación explícita de la disipación de energía a través de cambios microestructurales. Tal concepto tiene su fundamento en la teoría termo-mecánica ${ }^{[55 \text { y } 67]}$ en términos de funciones complementarias que representan la tasa de producción de entropía mediante conducción de calor y mediante cambios en la entropía interna causados por procesos microestructurales. A pesar de todas las críticas recibidas, está claro que el factor clave que decide el comportamiento dinámico de materiales durante un conformado en caliente es el parámetro $m$ que, en términos físicos simples, decide el mecanismo (o ruta) que el material elige constitutivamente bajo determinadas condiciones de conformado (p.ej., temperatura y velocidad de deformación). Dicho mecanismo será el camino "más corto" que el material debe seguir para disipar energía de la manera más eficiente posible, y que no es siempre el más "seguro" desde el punto de vista microestructural. Por lo tanto, en un proceso de conformado en caliente, los mecanismos de disipación de energía más eficientes no son, necesariamente, los más seguros ya que estos podrían ser causantes de daños microestructurales y roturas. También, dependiendo de las condiciones impuestas en el conformado en caliente, el sistema puede elegir un único mecanismo (determinista) o una combinación de dos o más mecanismos (probabilística) que tienen un valor característico del parámetro $m$.

Prasad añade que si bien la formulación del DMM no se deriva directamente de las consideraciones de la mecánica clásica de medios continuos, no contradice ninguno de sus principios requeridos para una fluencia estable. Además, la mecánica de medios continuos en grandes deformaciones no ha sido capaz de dar ninguna explicación al origen de la viscoplasticidad en materiales conformados plásticamente, considerando, únicamente, el área por debajo de curva de la ecuación constitutiva. Según Prasad, Ziegler ha sido el único en explicar, en su libro "Consideraciones Termomecánicas"[67], el origen del comportamiento de los materiales en condiciones de "creep" en caliente.

\subsection{Análisis crítico de Narayana Murty: DMM modificado}

Motivados por la discusión científica desarrollada entre Montheillet y Prasad, varios investigadores ${ }^{[11}$, 63 y 68-76] llevaron a cabo diversos estudios con el fin de entender mejor el DMM. En estos estudios, se ha demostrado que para describir el comportamiento a fluencia de materiales no se puede utilizar, indiscriminadamente, la ley constitutiva potencial, tanto en dominios de bajas como de altas tensiones. Es bien conocido que la ley constitutiva potencial es válida, únicamente, en el análisis de las tensiones de estado estable en condiciones de conformado a bajos valores de tensiones ${ }^{[1 \text { y } 77-79]}$.

Asimismo, Narayana Murty et al..$^{[11}$ y 68$]$ reanalizaron el DMM y propusieron otra metodología basada en la obtención directa de la eficiencia de disipación de energía a través de una integración numérica, a cualquier tensión de fluencia:

$$
\eta=\frac{J}{J_{\max }}=\frac{P-G}{P / 2}=2 \frac{J}{P}=2\left(1-\frac{1}{\sigma \dot{\varepsilon}} \int_{0}^{\dot{\varepsilon}} \sigma d \dot{\varepsilon}\right)
$$

Para integrar la función $G$ es necesario introducir valores, a partir de $\dot{\varepsilon}=0$. Para resolver este problema la integral de $\mathrm{G}$ se divide en:

$$
\eta=2\left[1-\frac{1}{\sigma \dot{\varepsilon}}\left(\int_{0}^{\dot{\varepsilon}_{\min }} \sigma d \dot{\varepsilon}+\int_{\dot{\varepsilon}_{\min }}^{\dot{\varepsilon}} \sigma d \dot{\varepsilon}\right)\right]
$$

$\dot{\varepsilon}_{\min }$ representa el valor más bajo de la velocidad de deformación usado en los ensayos de conformado en caliente. Para valores de $\dot{\varepsilon}$ superiores a $\dot{\varepsilon}_{\text {min }}$, la integral $\int_{\dot{\varepsilon}_{\min }}^{\dot{\varepsilon}} \sigma d \dot{\varepsilon}$ se calcula usando valores experimentales de la tensión de fluencia, $\sigma$. Para velocidades de deformación más bajos, es bien conocido que la ecuación de fluencia sigue una ley potencial, $\sigma=K \cdot \dot{\varepsilon}^{m}$, y se obtiene una nueva expresión de $\eta$ : 


$$
\eta=2\left[1-\frac{1}{\sigma \dot{\varepsilon}}\left(\left(\frac{\sigma \dot{\varepsilon}}{m+1}\right)_{\dot{\varepsilon}=\dot{\varepsilon}_{\min }}+\int_{\dot{\varepsilon}_{\min }}^{\dot{\varepsilon}} \sigma d \dot{\varepsilon}\right)\right]
$$

Así, teniendo en cuenta esta última expresión, Narayana Murty et al..$^{[11 \text { y } 68]}$ consideran que la condición de estabilidad expresada en la ecuación (74.I) es incorrecta. Argumentan que a una temperatura y una deformación dada, el parámetro $m$ varia con $\dot{\varepsilon}$, pero si la evolución de tensión de fluencia sigue una ley potencial, $m$ se convierte en un parámetro totalmente independiente de $\dot{\varepsilon}$. Por lo tanto, la condición de estabilidad establecida en la ecuación (74.I) se reduce a, simplemente, $m>0$ para una supuesta estabilidad metalúrgica.

Para establecer una nueva condición de estabilidad, Narayana Murty et al. usaron la ecuación (80.I) para expresar la función $J$ en términos de $\sigma, \dot{\varepsilon}$, y $\eta$ :

$$
J=\frac{1}{2} P \eta=\frac{1}{2} \sigma \dot{\varepsilon} \eta
$$
como:

Sabiendo que $J=\int_{0}^{\sigma} \dot{\varepsilon} d \sigma$, se puede volver a expresar

$$
\frac{\partial J}{\partial \dot{\varepsilon}}=\frac{\partial \sigma}{\partial \dot{\varepsilon}} \dot{\varepsilon}=\sigma \frac{\partial \ln \sigma}{\partial \ln \dot{\varepsilon}}=m \sigma
$$

sustituyendo las ecuaciones (83.I) y (84.I) en la ecuación (69.I), se puede obtener la siguiente condición de estabilidad en términos de $m$ y $\eta$ :

$$
\frac{2 m}{\eta}-1>0 \text { o bien } 2 m>\eta
$$

Así, para una fluencia estable, $\eta<2$ y $0<m<1$.

La condición de estabilidad expresada en la ecuación (85.I) es válida para cualquier tipo de evolución de la curva $\sigma=f(\dot{\varepsilon})$, sea de tipo potencial o exponencial. Si la curva $\sigma=f(\dot{\varepsilon})$ sigue una ley potencial, la eficiencia de disipación de energía será $\eta=\frac{2 m}{m+1}$, que es siempre inferior a $2 m$, para y $0<m$ $\leq 1$, por lo tanto, la fluencia del material será estable. La aplicabilidad de esta condición ha sido verifica-

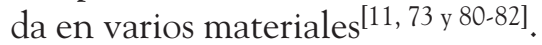

Para identificar los dominios estables en una fluencia plástica, Prasad ${ }^{[7}$ y 65] $u$ utilizó el co-contenido disipador, J, como función de disipación en el criterio de estabilidad de Ziegler expresado en la ecuación (68.I). Narayana Murty et al. ${ }^{[83]}$ consideraron interesante analizar la condición establecida en la ecuación (68.I), igualando la función de disipación, $D$, a
$P, G$ y J, respectivamente, y ver qué condiciones de estabilidad se obtienen.

Caso I: Cuando $D=P$

$$
\frac{d P}{d \dot{\varepsilon}}<\frac{P}{\dot{\varepsilon}} \Rightarrow \dot{\varepsilon} \frac{\partial \sigma}{\partial \dot{\varepsilon}}+\sigma \Rightarrow m<0
$$

es la condición de inestabilidad preferida por Montheillet et al. ${ }^{[64]}$, obtenida usando el criterio de inestabilidad de Ziegler.

Caso II: Cuando $D=G$

$$
\frac{d G}{d \dot{\varepsilon}}<\frac{G}{\dot{\varepsilon}} \Rightarrow \sigma \dot{\varepsilon}<G \Rightarrow P<G \Rightarrow J<0
$$

Caso III: Cuando $D=J$

$$
\frac{d J}{d \dot{\varepsilon}}<\frac{J}{\dot{\varepsilon}} \Rightarrow m \sigma<\frac{J}{\dot{\varepsilon}} \Rightarrow m P<J
$$

Cuando la tensión de fluencia sigue una evolución potencial sustituyendo el co-contenido, J, expresado en la ecuación (71.I), en las ecuaciones (87.I) y (88.I) se establece la condición $m<0$, obtenida en la ecuación (86.I).

Si la ecuación de fluencia no sigue una ley potencial, la condición $J<0$, de la ecuación (87.I) sigue siendo válida, incluso para valores de $m>0$, y la condición $J>m P$ de la ecuación (88.I), también, es válida para valores de $m$ que tienden a 0 , positivamente. En consecuencia, para una fluencia inestable para todos los materiales, la cuantificación de uno único rango de $m$ es imposible.

Es importante mencionar que los parámetros $\mathrm{J} / \mathrm{P}$ $(\equiv \eta / 2)$ y $m$ son parámetros adimensionales. Para obtener el parámetro $J$ se debe integrar $\sigma$ con respecto a $\dot{\varepsilon}$, mientras que para obtener el coeficiente $m$ es necesario una diferenciación de $\sigma$ con respecto a $\dot{\varepsilon}$. Además, el parámetro $J$ describe el comportamiento microestructural global de un material durante un proceso de conformado, mientras que el coeficiente $m$ proporciona información local de la sensibilidad que tiene el material a una velocidad de deformación dada. Por ello, sería más apropiado utilizar la condición de la ecuación (88.I) porque da valores negativos de $m(m<0)$ cuando $J<0$. Cuando $J>0$ y $m<0$ o tiende a ser igual a 0 , la condición establecida en la ecuación (88.I) permite obtener una mejor identificación de las regiones de inestabilidad plástica en los mapas de procesado. Cabe resaltar que la 
condición de estabilidad (85.I) puede obtenerse a partir de la ecuación (88.I), remplazando el parámetro $J$ en términos de la eficiencia de disipación de energía, $\eta$ (ecuación (83.I)).

\section{Agradecimientos}

Uno de los autores, Anas Al Omar, desea agradecer a la CICYT (España) la financiación económica a través del proyecto DPI2007-63665.

\section{REFERENCIAS}

[1] J.J. Jonas, C.M. Sellars y W.J. McG. Tegart, Metall. Rev. 14 (1969) 1-12.

[2] P.W. Lee y H.A. Kuhn, Metall. Trans. A 4 (1973) 969-974.

[3] R. Raj, Metall.Trans. A 12 (1981) 1.089-1.097.

[4] H.J. Frost y M.F. Ashby, Deformation-mechanism maps, caps. 1-5, Ed. Pergamon Press, Oxford, Inglaterra, 1982.

[5] Y.V.R.K. Prasad, H.L. Gegel, S.M. Doraivelu, J.C. Malas, J.T. Morgan, K.A. Lark y D.R. Barker, Metall.Trans. A 15 (1984) 1.883-1.892.

[6] G.E. Dieter, Metals Handbook, ASM, Metals Park, Ohio, 14A (1987) 363-387.

[7] Y.V.R.K. Prasad, Ind. J. Technol. 28 (1990) 435 451.

[8] Y.V.R.K. Prasad y N. Ravichandran, Bull. Mater. Sci. 14 (1991) 1.241-1.248.

[9] Y.V.R.K. Prasad y S. Sasidhara, Hot Working Guide: A Compendium of Processing Maps, ASM International, Materials Park, Ohio, 1997.

[10] Y.V.R.K. Prasad y T. Seshacharyulu, Int. Mater. Rev. 43 (1998) 243-258.

[11] S.V.S. Narayana, B. Nageswara y B.P. Kash, Int. Mater. Rev. 45 (2000) 15-26.

[12] A. Al Omar y J.M. Prado, Rev. Metal. Madrid 33 (1997) 89-100.

[13] I. Rieiro, A. Fernández, A. Martínez y M. Carsí, Rev. Metal. Madrid 34 (1998) 355-366.

[14] M. Carsí, R. Allende, F. Peñalba, J.A. Jiménez y O.A. Ruano, Steel Res.Int. 75 (2004) 26-32.

[15] I. Rieiro, M. Cars> y O.A. Ruano, Mater. Sci. Technol. 25 (2009) 995-1.002.

[16] S.L. Semiatin y J.J. Jonas, Formability and Workability of Metals: Plastic Instability and Flow Localization, ASM, Metals Park, Ohio, 1984.

[17] R.C. Batra y C.H. Kim, Int. J. Plast. 8 (1992) 425-452.

[18] S. L. Semiatin, G.D. Lahoti, y S.I. Oh, Material Behavior Under High Stress and Ultra high Loading Rates, 29th Sagamore Army Mater. Res. Conf.
Proc., Ed. J. Mescall y V. Weiss, Plenum Press, Nueva York, EE.UU., 1982, pp. 119-159.

[19] A. J. Bedford, A. L. Wingrove y K. R. L. Thompson, J. Aust. Inst. Met. 19 (1974) 61-73.

[20] G.B. Olsen, J.F. Mescall y M. Azrin, Shock Waves and High strain Rate Phenomena in Metals, Ed. M.A. Meyers y L.E. Murr, Plenum Press, Nueva York, EE.UU., 1980, pp. 221-247.

[21] H.C. Rogers, Ann. Rev. Mater. Sci. 9 (1979) 283-311.

[22] Y. Liu, R. Hu, J. Li, H. Kou, H. Li, H. Chang y H. Fu, Mater. Sci. Eng. A 508 (2009) 141-147.

[23] L.J. Huang, L. Geng, A.B. Li, X.P. Cui, H.Z. Li y G.S. Wang, Mater. Sci. Eng. A 505 (2009) 136-143.

[24] Z. Marciniak y K. Kuczynski, Int. J. Mech. Sci. 9 (1967) 609-620.

[25] Z. Marciniak, K. Kuczynski y T. Pokora, Int. J. Mech. Sci. 15 (1973) 789-805.

[26] J. J. Jonas, R. A. Holt y C.E. Coleman, Acta Metall. 24 (1976) 911 -918.

[27] J.J. Jonas y B. Baudelet, Acta Metall. 25 (1977) 4350.

[28] D. Campbell, J. Mech. Phys. Solids 15 (1967) 359-370.

[29] A.S. Argon Avery, The inhomogeneity of plastic deformation, ASM International, Materials Park, Ohio, 1973.

[30] A. Considère, Ann. Ponts Chaussées 9 (1885) 574-575.

[31] W.A. Backofen, I.R. Turner y D.H. Avery, Trans. ASM 57 (1964) 980-990.

[32] E.W. Hart, Acta Metall. 15 (1967) 351-355.

[33] S.L. Semiatin y G. D. Lahoti, Metall. Trans. A 12 (1981) 1.705-1.717.

[34] M. Aghaie-Khafri y N. Golarzi, Mater. Sc. Eng. A 486 (2008) 641-647.

[35] S.L. Semiatin y G.D. Lahoti, Metall. Trans. A 13 (1982) 275-288.

[36] A.R. Salehi, S. Serajzadeh y N. Yazdipour, Mater. Chem. Phys. 101(2007) 153-157

[37] H.L. Gegel, J.C. Malas, S.M. Doraivelu y V.A. Shende, Metals Handbook vol. 14,, ASM, Metals Park, 1987, pp. 417-438.

[38] H.L. Gegel, J.C. Malas, S.M. Doraivelu, J.M Alexander y J.S. Gunasekera, Proc. Adv. Technol. Plasticity, vol. 11, Ed. K. Lange, Springer-Verlag, Londres, Inglaterra, 1987, pp. 1.243-1.249.

[39] J.M. Alexander, Modelling of hot deformation of steels, Ed. J.G. Lenard, Springer-Verlag, Berlin, Alemania, 1989, pp. 101-115.

[40] H. Ziegler, Progress in solid mechanica, vol. 4, Ed. I.N. Sneddon y R. Hill, Nueva York, EE. UU., 1963, pp. 93-193. 
[41] P.E. Wellstead, Introduction to Pysical Systems Modelling, Ed. Academic Press, Londres, Inglaterra, 1979.

[42] I. Prigogene, Science 201 (1978) 777-787.

[43] D. Cai, L. Xiong, W. Liu, G. Sun y M. Yao, Mater. Des. 30 (2009) 921-925.

[44] L.J. Huang, L. Geng, A.B. Li, X.P. Cui, H.Z. Li y G.S. Wang, Mater. Sci. Eng. A 505 (2009) 136-143.

[45] J. Luo, M. Li, H. Li y W. Yu, Mater. Sci. Eng. A 505 (2009) 88-95.

[46] J. Luo, M. Li, W. Yu y H. Li, Mater. Sci. Eng. A 504 (2009) 90-98.

[47] M.Q. Li y W.F. Zhang, Mater. Sci. Eng. A 502 (2009) 32-37.

[48] Y.V.R.K. Prasad, K.P. Rao, N. Hort y K.U. Kainer, Mater. Sci. Eng. A 502 (2009) 25-31.

[49] F. Bakkali El Hassani, A. Chenaoui, R. Dkiouak, L. Elbakkali y A. Al Omar, J. Mater. Process. Technol. 199 (2008)140-149.

[50] A. Al Omar, Tesis Doctoral, Univ. Politécnica de Cataluña, 1996.

[51] A. Al Omar, J.M. Cabrera Y J.M. Prado, Scr. Mater.34 (1996) 1303-1308.

[52] A. Al Omar, J.M. Cabrera Y J.M. Prado, Rev. Metal. Madrid 33 (1997) 153-160

[53] I. Prigogene, Science 201 (1978) 777-787.

[54] C. Basaran y S. Nie, Int. J. Damage Mech. 13 (2004) 205-223.

[55] L.E. Malvern, Introduction to the Mechanics of Continuum Medium, Ed. Prentice-Hall, New Jersey, EE. UU., 1969.

[56] J. Lasalla y L. Solomon, Stability by Liapunov's direct method, Ed. Academica Press. Boston, EE. UU., 1987.

[57] D.L. Schultz y J.L. Melsa, State Functions and Linear Control Systems, Ed. McGraw-Hill, 1967.

[58] H.L. Gegel, Computer simulation in material science, Ed. R.J. Arsenault et al., ASM, Metals Park, vol. 14, 1987, pp. 291-344.

[59] A.K.S. Kalyan, MS Thesis, Indian Institute of Science, Bangalore, India, 1987.

[60] T. Rajagopalachary y V.V. Kutumbarao, Scr. Mater. 35 (1996) 311-316.

[61] V.V. Kutumbarao y T. Rajagopalachary, Bull. Mater. Sci. 19 (1996) 677-698.
[62] R. Hill, J. Mech. Phys. Solids 35 (1987) 23-33.

[63] S.V.S. Narayana y B. Nageswara, Bull. Mater. Sci. 22 (1999) 9-10.

[64] F. Montheillet, J. J. Jonas y K.W. Neale, Metall. Trans. A 27 (1996) 232-235.

[65] Y.V.R.K. Prasad, Metall. Trans. A 27 (1996) 235-236.

[66] Y.V.R.K. Prasad, J. Mater. Eng. Performance 12 (2003) 638-345.

[67] H. Ziegler, Thermomechanics, $2^{\text {nd }}$ ed., Ámsterdam, Holanda, 1983.

[68] S.V.S. Narayana, M.S. Sarma y B. Nageswara, Metall. Trans. A 28(1997) 1.581-1.582.

[69] E.S. Puchi y M.H. Staia, Metall. Trans. A 26, (1995) 2.895-2.910.

[70] E.S. Puchi y M.H. Staia, Metall. Trans. A 29 (1998) 2.345-2.359.

[71] S. Ghosh, Metall. Trans. A 31 (2000) 2.9732.974 .

[72] S. Ghosh, Metall. Trans. A 33 (2002) 1.569. 1.572 .

[73] C. Poletti, H.P. Degischer, S. Kremmer y W. Marketz, Mater. Sci. Eng. A 486 (2008) 127 137.

[74] C. Poletti, H. Dieringa y F. Warchomicka, Mater. Sci. Eng. A 516 (2008) 138-147.

[75] J. Liu, Z. Cui y C. Li, J. Mater. Process. Technol. 205 (2008) 497-505

[76] G. Meng, B. Li, H. Li, H. Huang y Z. Nie, Mater. Sci. Eng. A 517 (2009) 132-137.

[77] J.M. Cabrera, A. Al Omar y J.M. Prado, Rev. Metal. Madrid 33 (1997) 215-228.

[78] J.M. Cabrera, A. Al Omar, J.J. Jonas y J.M. Prado, Metall. Trans. A 28 (1997) 2.233-2.244.

[79] A. Al Omar, J.M. Cabrera y J.M. Prado, Rev. Métall. 5 (1998) 589-597

[80] S.V.S. Narayana, B. Nageswara y B.P. Kashyap, J. Mater. Process. Technol. 166 (2005) 268-278.

[81] S.V.S. Narayana, B. Nageswara y B.P. Kashyap, J. Mater. Process. Technol. 166 (2005) 279-285.

[82] S.V.S. Narayana, B. Nageswara y B.P. Kashyap, Compos. Sci. Technol. 63 (2003) 119-135.

[83] S.V.S. Narayana y B. Nageswara, J. Mater. Sci. Lett. 18 (1999) 1.757-1758. 